\title{
COVERING THEOREMS WITH APPLICATIONS TO SOME QUESTIONS ON NORMAL ORDERS
}

\author{
BY \\ W. R. EMERSON
}

0. Introduction. In [1] the question of the asymptotic distribution of squarefree integers in "moving" intervals is considered, and a proof is given of

THEOREM A. If $\phi: N \rightarrow P=\{$ positive real numbers $\}$ is any monotone function satisfying

$$
\lim _{n \rightarrow \infty} \phi(n)=+\infty,
$$

then $Q_{\phi}(n)=Q(n, n+\phi(n))$ has normal order $6 \phi(n) / \pi^{2}$, where $Q(u, v)$ is the number of squarefree integers $q$ satisfying $u<q \leqq v$.

In turn, the proof of Theorem $A$ is made to depend on the following general result of [1]:

TheOREM $\mathrm{A}^{\prime}$. Let $A=\left\{a_{v}\right\}$ be any sequence of integers whose cumulant $A(u, v)$ $=\sum_{u<a_{v} \leqq v} 1$ satisfies

$$
A(x) \doteqdot A(0, x)=\alpha x+o\left(x^{1 / 2}\right)
$$

for some positive constant $\alpha$, and let $\phi: N \rightarrow P$ be a monotone function satisfying $\lim _{n \rightarrow \infty} \phi(n)=+\infty$ and

$$
\limsup _{n \rightarrow+\infty} \frac{A(n, n+\phi(n))}{\alpha \phi(n)} \leqq 1 .
$$

Then $A_{\phi}(n)=A(n, n+\phi(n))$ has normal order $\alpha \phi(n)$.

That the sequence $Q$ of squarefree integers satisfies the asymptotic estimate $\left(^{*}\right)$ is a result roughly on the level of the Prime Number Theorem, while condition (**) is easily verified for $Q$ if $\phi(n)$ is any function tending to $+\infty$ with $n$ (Lemma 2.1 of [1]). The proof of Theorem $A^{\prime}$ uses a method of inversion of a double summation and depends rather heavily on the estimate $\left({ }^{*}\right)$ to control the error so introduced. Furthermore the authors state that they were unable to relax the monotonicity assumption on $\phi$ in Theorem $\mathrm{A}$ using the technique at hand.

We first prove (Theorem 1) that the monotonicity hypothesis on $\phi$ in Theorem A may be eliminated by a wholly elementary argument utilizing the validity of $\left({ }^{* *}\right)$ if $A=Q$ for all such monotone $\phi$ (Lemma 2.1 of [1]). We then reconsider Theorem $\mathrm{A}^{\prime}$ in the general context where $\left({ }^{* *}\right)$ is known to be valid for only a single $\phi$. We

Received by the editors April 15, 1969.

Copyright (C) 1969, American Mathematical Society 
then show by counterexample (Theorem 2) that if $\phi$ is not assumed monotone, hypothesis $\left({ }^{* *}\right)$ and even a great sharpening of $(*)$ do not necessarily imply that $A_{\phi}$ has normal order $\alpha \phi$. On the other hand, if $\phi$ is assumed monotone and $\left(^{*}\right)$ is replaced by merely $A(x) \sim \alpha x$ then the conclusion of Theorem $\mathrm{A}^{\prime}$ remains valid (Theorem 4). The totally elementary proof of this result does not use the methods on [1], but rather proceeds via an investigation of a combinatorial covering problem in $N$ (Theorem 3).

This technique is then extended to treat more general asymptotic analogues of Theorem $\mathrm{A}^{\prime}$ (Theorem 6) by suitably generalizing the requisite combinatoric argument (Theorem 5). Also, an example indicates certain natural limitations to this extension procedure. Finally, a continuous analogue of these questions is considered and a sketch of its totally analogous resolution is given (Theorems 7 and 8).

I should like to thank Professor H. L. Shapiro for suggesting this problem and R. L. Levine for many useful conversations.

I. Asymptotic bounds for all $\phi$. In this section we show that the monotonicity hypothesis in Theorem $\mathrm{A}$ is not necessary. We consider the following context:

$A: P \rightarrow P$ is any nondecreasing function tending to infinity; $A(x, y) \doteqdot A(y)-A(x)$; if $\phi: P \rightarrow P, A_{\phi}(x)=A(x, x+\phi(x))$.

Note. If $A=\left\{a_{v}\right\}$ is any infinite sequence of positive numbers tending to infinity then the cumulant function $A(x) \doteqdot \sum_{a_{v} \leqq x} 1$ is such a function. We now prove:

LEMmA 1. A as above, $\alpha>0$. Then the following conditions are equivalent:

$$
\limsup _{v \rightarrow \infty} \frac{A\left(x_{v}, x_{v}+y_{v}\right)}{y_{v}} \leqq \alpha \quad \begin{aligned}
& \text { for all sequences }\left\{x_{v}\right\},\left\{y_{v}\right\} \subseteq P \\
& \text { tending to }+\infty
\end{aligned}
$$

$$
\limsup _{n \rightarrow \infty} \frac{A(n, n+\phi(n))}{\phi(n)} \leqq \alpha \quad \text { for all } \phi: N \rightarrow P \text { tending to }+\infty \text {. }
$$

$$
\begin{array}{ll}
\limsup _{n \rightarrow \infty} \frac{A(n, n+\phi(n))}{\phi(n)} \leqq 0 & \begin{array}{l}
\text { for all } \phi: N \rightarrow P \text { tending } \\
\text { monotonely to }+\infty .
\end{array}
\end{array}
$$

(iv) $\quad \limsup _{y \rightarrow+\infty}\left(\limsup _{x \rightarrow+\infty} \frac{A(x, x+y)}{y}\right) \leqq \alpha$.

$$
\limsup _{x, y \rightarrow+\infty} \frac{A(x, x+y)}{y} \leqq \alpha .
$$

Proof. (i) $\Rightarrow$ (ii) $\Rightarrow$ (iii) is trivial. To see that (iii) $\Rightarrow$ (iv), assume (iv) violated, i.e. for some $\varepsilon>0$

$$
\limsup _{y \rightarrow+\infty}\left(\limsup _{x \rightarrow+\infty} \frac{A(x, x+y)}{y}\right) \geqq \alpha+\varepsilon .
$$

Consequently, there exist sequences $\left\{x_{v}\right\},\left\{y_{v}\right\}$ in $P$ tending to infinity for which

$$
A\left(x_{v}, x_{v}+y_{v}\right) / y_{\nu}>\alpha+\varepsilon / 2, \quad \text { all } \nu \in N,
$$


and without loss of generality we may assume $x_{v+1}>x_{v}+1, y_{v+1}>y_{v}+1$. Now set $n_{v}=\left[x_{v}\right], \phi\left(n_{v}\right)=\left(x_{v}-\left[x_{v}\right]\right)+y_{v}=x_{v}-n_{v}+y_{v}$, noting that $\phi\left(n_{v}\right)$ is increasing in $v$. Now

$$
\begin{aligned}
A\left(n_{v}, n_{v}+\phi\left(n_{v}\right)\right) & =A\left(n_{v}, x_{v}+y_{v}\right) \geqq A\left(x_{v}, x_{v}+y_{v}\right)>(\alpha+\varepsilon / 2) y_{v} \\
& =(\alpha+\varepsilon / 2)\left(\phi\left(n_{v}\right)-\left\{x_{v}-\left[x_{v}\right]\right\}\right) .
\end{aligned}
$$

Therefore if $\phi$ is extended arbitrarily to a monotone function on all of $N$, we have by (1.1)

$$
\begin{aligned}
\limsup _{n \rightarrow+\infty} \frac{A(n, n+\phi(n))}{\phi(n)} & \geqq \limsup _{v \rightarrow+\infty} \frac{A\left(n_{v}, n_{v}+\phi\left(n_{v}\right)\right)}{\phi\left(n_{v}\right)} \\
& \geqq \limsup _{v \rightarrow+\infty}\left(\alpha+\frac{\varepsilon}{2}\right) \frac{\left(\phi\left(n_{v}\right)-\left\{x_{v}-\left[x_{v}\right]\right\}\right)}{\phi\left(n_{v}\right)}=\alpha+\frac{\varepsilon}{2},
\end{aligned}
$$

in violation of (iii).

To prove that (iv) $\Rightarrow(\mathrm{v})$, we must show that given any $\varepsilon>0$ there exists a $w=w(\varepsilon)$ such that

$$
A(x, x+y) / y<\alpha+\varepsilon, \quad \text { all } x, y \geqq w .
$$

To this end, fix any $n \in N$. Then by (iv) for all $y \geqq y_{0}=y_{0}(n)$

$$
\limsup _{x \rightarrow+\infty} \frac{A(x, x+y)}{y}<\left(\alpha+\frac{1}{n}\right) \text {. }
$$

Consequently, to each $y \geqq y_{0}$ there corresponds an $x_{0}=x_{0}(y)$ such that

$$
A(x, x+y) / y<(\alpha+2 / n) \text { for } x \geqq x_{0} .
$$

Now set

$$
w=\max \left\{2 y_{0}, x_{0}\left(y_{0}\right), x_{0}\left(y_{0}+(1 / n) y_{0}\right), x_{0}\left(y_{0}+(2 / n) y_{0}\right), \ldots, x_{0}\left(y_{0}+y_{0}\right)\right\},
$$

and let $x, y \geqq w$. We may then write

$$
y=q y_{0}+y^{\prime} \quad \text { where } q \in N \text { and } y_{0} \leqq y^{\prime}<2 y_{0} .
$$

Now by definition of $A(x, y)$

$$
A(x, x+y)=A\left(x, x+y^{\prime}\right)+\sum_{0 \leqq i<q} A\left(x+y^{\prime}+i y_{0}, x+y^{\prime}+(i+1) y_{0}\right) .
$$

Now assume $y_{0}+((r-1) / n) y_{0} \leqq y^{\prime}<y_{0}+(r / n) y_{0}, 1 \leqq r \leqq n$. Then (1.3), (1.4) and the definition of $w$ yield

$$
\begin{aligned}
A(x, x+y) & \leqq A\left(x, x+y_{0}+(r / n) y_{0}\right)+\sum_{0 \leqq i<q} A\left(x+y^{\prime}+i y_{0}, x+y^{\prime}+(i+1) y_{0}\right) \\
& \leqq(\alpha+2 / n)\left\{\left(y_{0}+(r / n) y_{0}\right)+q y_{0}\right\} \\
& =(\alpha+2 / n)\left\{y+\left(y_{0}+(r / n) y_{0}-y^{\prime}\right)\right\} \leqq(\alpha+2 / n)\left(y+y_{0} / n\right) \\
& \leqq(\alpha+2 / n)(y+y / n)=(\alpha+2 / n)(1+1 / n) y .
\end{aligned}
$$


Now clearly if $n \in N$ is large enough and $\varepsilon>0$ is fixed $(\alpha+2 / n)(1+1 / n)<\alpha+\varepsilon$, and therefore (1.2) is verified.

Finally, (v) $\Rightarrow$ (i) is immediate and consequently (i)-(v) are equivalent.

We now prove

THEOREM 1. Assume for some $\alpha>0$ that $A$ satisfies:

1. $A(x) \sim \alpha x$.

2. Any (hence all) of the conditions of Lemma 1 (for this same value of $\alpha$ ).

Then if $\phi: N \rightarrow P$ is any function tending to $+\infty, A_{\phi}(n)=A(n, n+\phi(n))$ has normal order $\alpha \phi(n)$.

Comment. This is the promised "elementary" strengthening of Theorem A in [1] since the cumulant function $Q(x)=\sum_{q \in Q: q \leqq x} 1$ of the squarefree integers $Q$ is easily shown to satisfy 1 , for $\alpha=6 / \pi^{2}$ and also condition (iv) of Lemma 1 for this value of $\alpha$ (Lemma 2.1 of [1]).

Proof. By contradiction. Assume $A_{\phi}(n)=A(n, n+\phi(n))$ does not have normal order $\alpha \phi(n)$, where $\phi: N \rightarrow P$ is some function tending to $+\infty$. This means there exists an $\varepsilon>0$ such that

$$
A^{*}(\varepsilon)=\left\{n \in N:\left|A_{\phi}(n)-\alpha \phi(n)\right|>\varepsilon \phi(n)\right\}
$$

does not have asymptotic density zero, i.e.

$$
\limsup _{x \rightarrow+\infty} \sum_{n \in A^{*}(\varepsilon): n \leqq x} 1 / x=\delta>0 .
$$

Now by hypothesis 2 (using condition (ii) of Lemma 1) there exists an $n_{0}=n_{0}(\varepsilon)$ such that

$$
\frac{A_{\phi}(n)}{\phi(n)}=\frac{A(n, n+\phi(n))}{\phi(n)}<\alpha+\frac{\varepsilon}{2} \quad \text { for } n \geqq n_{0} .
$$

Consequently by (1.5)

$$
A_{\phi}(n)<(\alpha-\varepsilon) \phi(n) \quad \text { for } n \in A^{*}(\varepsilon), n \geqq n_{0} .
$$

By virtue of (1.6), given any $n_{1} \geqq n_{0}$ we may find arbitrarily large $m>n_{1}$ for which

$$
\sum_{n \in A^{*}(\varepsilon): n_{1} \leqq n \leqq m} 1>[(\delta / 2) m] .
$$

We now consider the interval $I=\left[n_{1}, m\right]$ for any such $n_{1}, m$, and obtain a lower bound for the sum of the lengths of an appropriately chosen disjoint family of intervals $I_{n}=[n, n+\phi(n)]$ contained in $I$ with every such $n \in A^{*}(\varepsilon)$. To this end, let

$$
\phi_{*}=\min \{\phi(n): n \in I\}, \quad \phi^{*}=\max \{\phi(n): n \in I\} .
$$

Consequently, $I_{n}=[n, n+\phi(n)] \subseteq I$ for every $n \in I$ which satisfies $n \leqq m-\phi^{*}$. Furthermore each interval $I_{n}$ contains at most $\phi(n)+1$ integer points. Now to construct the desired family of intervals we proceed recursively: let $n^{(1)}$ be the smallest integer in $A^{*}(\varepsilon) \cap I$, and $I_{n^{(1)}}$ the first interval; let $n^{(2)}$ be the smallest 
integer in $A^{*}(\varepsilon)$ larger than $n^{(1)}+\phi\left(n^{(1)}\right)$, and $I_{n^{(2)}}$ be the second interval. We continue recursively in this fashion until we reach the first $n^{(k)}$ such that $I_{n}(k) \nsubseteq I$. Then clearly the intervals $I_{n}^{(j)}, 1 \leqq j<k$, are disjoint and contained in $I$, each $n^{(j)} \in A^{*}(\varepsilon)$, and any point of $A^{*}(\varepsilon) \cap I$ not contained in one of these intervals must be in $\left(m-\phi^{*}, m\right]$. Therefore by (1.9)

$$
\frac{\delta}{2} m<\phi^{*}+\sum_{1 \leqq j<k}\left(\phi\left(n^{(j)}\right)+1\right) .
$$

Furthermore, $k$ satisfies

$$
k \leqq m / \phi_{*}
$$

since each $I_{n}^{(j)}$ is of length $\left|I_{n^{(j)}}\right| \geqq \phi_{*}$.

Now consider the nontrivial intervals among

$$
\left(n_{1}, n^{(1)}\right],\left(n^{(1)}+\phi\left(n^{(1)}\right), n^{(2)}\right], \ldots,\left(n^{(k-1)}+\phi\left(n^{(k-1)}\right), m\right],
$$

and call them $J_{1}, \ldots, J_{r}$, where $r \leqq k$. Then divide these intervals into two subsets depending on their length:

$$
\mathscr{J}^{+}=\left\{J_{i}:\left|J_{i}\right| \geqq\left|\phi_{*}\right|^{1 / 2}\right\}, \quad \mathscr{J}^{-}=\left\{J_{i}:\left|J_{i}\right|<\left|\phi_{*}\right|^{1 / 2}\right\},
$$

$\left(\left.\right|^{*} \mid\right.$ always denotes length/measure). Next replace each $J_{i}$ in $\mathscr{J}^{-}$by $J_{i}^{\prime}$, the interval with the same left-hand "endpoint" as $J_{i}$ but of length $\left|\phi_{*}\right|^{1 / 2}$, and leave each $J_{i}=J_{i}^{\prime} \in \mathscr{J}^{+}$unchanged. Then clearly by (1.12)

$$
\sum_{1 \leqq i \leqq r}\left|J_{i}^{\prime}\right| \leqq \sum_{1 \leqq i \leqq r}\left|J_{i}\right|+k\left|\phi_{*}\right|^{1 / 2} \leqq \sum_{1 \leqq i \leqq r}\left|J_{i}\right|+\frac{m}{\left|\phi_{*}\right|^{1 / 2}}
$$

By construction $\left(n_{1}, m\right] \subseteq \bigcup_{1 \leqq j<k} I_{n}{ }^{(j)} \bigcup_{1 \leqq j \leqq r} J_{i}{ }^{\prime}$, and consequently,

$$
A\left(n_{1}, m\right) \leqq \sum_{1 \leqq j<k} A_{\phi}\left(n^{(j)}\right)+\sum_{1 \leqq j \leqq r} A\left(x_{i}, y_{i}\right)
$$

where $J_{i}^{\prime}=\left(x_{i}, y_{i}\right]$. We are now almost ready for the final estimate, but first we shall show that there is no loss in generality if we assume

$$
\phi(n)=o(n) .
$$

For by hypothesis $1, A(x) \sim \alpha x$ is equivalent to $A(x)=\alpha x+e(x)$, where $|e(x)|<\sigma(x) x$ for some appropriate function $\sigma$ tending monotonely to zero as $x \rightarrow+\infty$. Now consider

$$
\tilde{\phi}(n)=\min \left(\phi(n),(\sigma(n))^{1 / 2} n\right)=o(n) .
$$

Then claim for any $\varepsilon>0$, if we set

and

$$
A^{*}(\varepsilon)=\left\{n \in N:\left|A_{\phi}(n)-\alpha \phi(n)\right|>\varepsilon \phi(n)\right\}
$$

$$
\tilde{A}^{*}(\varepsilon)=\left\{n \in N:\left|A_{\tilde{\phi}}(n)-\alpha \tilde{\phi}(n)\right|>\varepsilon \tilde{\phi}(n)\right\}
$$

then $A^{*}(\varepsilon) \sim \tilde{A}^{*}(\varepsilon)$ is finite. This justifies the simplifying hypothesis (1.16) since 
$\tilde{A}^{*}(\varepsilon)$ having asymptotic density zero implies $A^{*}(\varepsilon)$ does also. Now $n \in A^{*}(\varepsilon) \sim \tilde{A}^{*}(\varepsilon)$ implies

$$
\left|A_{\phi}(n)-\alpha \phi(n)\right|>\varepsilon \phi(n), \quad \tilde{\phi}(n)=(\sigma(n))^{1 / 2} n<\phi(n) .
$$

But

$$
\begin{aligned}
\left|A_{\phi}(n)-\alpha \phi(n)\right| & =|A(n+\phi(n))-A(n)-\alpha \phi(n)| \\
& =|\alpha(n+\phi(n))-\alpha n-\alpha \phi(n)+e(n+\phi(n))-e(n)| \\
& =|e(n+\phi(n))-e(n)| \leqq|e(n+\phi(n))|+|e(n)| \\
& <\sigma(n)(n+\phi(n)+n)<2 \sigma(n)(n+\phi(n)) .
\end{aligned}
$$

Consequently, (1.17) and (1.18) yield $\varepsilon \phi(n)<2 \sigma(n)(n+\phi(n))$, or upon transposing

$$
(\varepsilon-2 \sigma(n)) \phi(n)<2 \sigma(n) n .
$$

Hence by (1.17) and (1.19), if $n$ is large enough to insure $2 \sigma(n)<\varepsilon / 2,(\varepsilon / 2)(\sigma(n))^{1 / 2} n$ $<(\varepsilon / 2) \phi(n)<(\varepsilon-2 \sigma(n)) \phi(n)<2 \sigma(n) n$, which in turn implies $\varepsilon / 4<(\sigma(n))^{1 / 2}$, and therefore $n$ is bounded which proves our assertion.

Finally, fix any $\theta, 0<\theta<\varepsilon$ and by Lemma (1.v) find $w=w(\theta)$ such that

$$
A(x, x+y)<(\alpha+\theta) y \quad \text { if } x, y \geqq w(\theta) .
$$

Now assume $n_{1}$ is choosen so large that

$$
n_{1} \geqq w(\theta), \quad \phi(n) \geqq(w(\theta))^{2} \quad \text { all } n \geqq n_{1} .
$$

Consequently by (1.8), (1.15) and (1.20)

$$
A\left(n_{1}, m\right) \leqq\left(\sum_{1 \leqq j<k} \phi\left(n^{(j)}\right)\right)(\alpha-\varepsilon)+\left(\sum_{1 \leqq i \leqq r}\left|J_{i}^{\prime}\right|\right)(\alpha+\theta) .
$$

Now by (1.11), (1.12),

$$
\frac{\delta}{2} m-\phi^{*}-\frac{m}{\phi_{*}} \leqq \sum_{1 \leqq j<k} \phi\left(n^{(j)}\right) \doteqdot \sum
$$

and since

$$
\sum+\sum_{1 \leqq i \leqq r}\left|J_{i}\right|=m-n_{1},
$$

(1.21), (1.22) and (1.14) imply

$$
\begin{aligned}
A\left(n_{1}, m\right) & \leqq\left(\sum\right)(\alpha-\varepsilon)+\left(m-n_{1}-\sum+\frac{m}{\left|\phi_{*}\right|^{1 / 2}}\right)(\alpha+\theta) \\
& =\left(m-n_{1}\right)(\alpha+\theta)-(\varepsilon+\theta) \sum+\frac{m}{\left|\phi_{*}\right|^{1 / 2}}(\alpha+\theta) \\
& \leqq\left(m-n_{1}\right)(\alpha+\theta)-(\varepsilon+\theta)\left(\frac{\delta}{2} m-\phi^{*}-\frac{m}{\phi_{*}}\right)+\frac{m}{\left|\phi_{*}\right|^{1 / 2}}(\alpha+\theta) \\
& \leqq m\left(\alpha+\theta-\frac{(\varepsilon+\theta) \delta}{2}+\varepsilon \frac{\phi^{*}}{m}+\frac{\varepsilon}{\phi_{*}}+\frac{(\alpha+\theta)}{\left|\phi_{*}\right|^{1 / 2}}\right) \\
& =m\left(\alpha-\frac{\varepsilon \delta}{2}+\theta-\frac{\theta \delta}{2}+\varepsilon \frac{\phi^{*}}{m}+\frac{\varepsilon}{\phi_{*}}+\frac{(\alpha+\theta)}{\left|\phi_{*}\right|^{1 / 2}}\right) .
\end{aligned}
$$


Now as both $n_{1}$ and $m>n_{1}$ tend to $+\infty$ through admissible values (see (1.9)) for which $m>n_{1}$

$$
\varepsilon \frac{\phi^{*}}{m}, \quad \frac{\varepsilon}{\phi_{*}}, \quad \frac{\alpha+\theta}{\left|\phi_{*}\right|^{1 / 2}} \rightarrow 0 .
$$

Furthermore, for $\theta$ sufficiently small $\varepsilon \delta / 4>\theta-\theta \delta / 2$, and consequently for admissible $m>n_{1}$ both sufficiently large $A\left(n_{1}, m\right) \leqq m(\alpha-\varepsilon \delta / 5)$, which contradicts hypothesis 1 if $m \rightarrow+\infty$ through admissible values (for fixed $n_{1}$ sufficiently large).

II. The analysis of a single $\phi$. We first show that the monotonicity assumption on $\phi$ in Theorem $A^{\prime}$ may not be wholly discarded, even upon strengthening the hypothesis $(*)$ :

THEOREM 2. Let $w$ be any function tending to $+\infty$. Then there exists a sequence $A=A(w)=\left\{a_{k}\right\}$ whose cumulant satisfies

$$
A(x)=\frac{1}{2} x+o(w(x))
$$

and a function $\phi: N \rightarrow P$ such that $\lim _{n \rightarrow \infty} \phi(n)=+\infty$, and

$$
\limsup _{n \rightarrow+\infty} \frac{A(n, n+\phi(n))}{\phi(n)} \leqq \frac{1}{2}
$$

whereas $A_{\phi}(n)=A(n, n+\phi(n))$ does not have normal order $\frac{1}{2} \phi(n)$. Moreover, $A_{\phi}(n)=0$ on a set of positive asymptotic density.

Comment. The choice of $\alpha=\frac{1}{2}$ is for technical convenience only, and an analogous construction shows the existence of such an $A^{(\alpha)}$ for any $\alpha, 0<\alpha<1$ (depending on $w$ also, of course). Furthermore, upon fixing this $A^{(\alpha)}$ a minor modification of our construction allows us to find appropriate $\phi: N \rightarrow P$ for which $A^{(\alpha)}(n, n+\phi(n))=0$ on a set of asymptotic density arbitrarily close to $1-\alpha$.

Proof. Divide $N$ into consecutive blocks $B_{i}$ of disjoint integers as follows: first $y_{1}$ blocks with 1 element, then $y_{2}$ blocks with 2 elements, then $y_{3}$ blocks with 3 elements, etc. where $y_{1}, y_{2}, y_{3}, \ldots$ are any positive integers for which

$$
w\left(y_{1}+2 y_{2}+\cdots+k y_{k}\right)>k^{2} \quad(k \in N),
$$

which is clearly possible if the $y_{i}$ are chosen sufficiently large since $w$ tends to $+\infty$. Furthermore, we may clearly assume $w$ tends monotonely to $+\infty$ without loss of generality. Now let $A$ be the union of the integers in the even indexed blocks,

$$
A=\bigcup_{j \in N} B_{2 j}
$$

Therefore condition (2.1) is satisfied by $A$ by virtue of (2.3). We next define $\phi$ :

(i) If $n \in B_{2 j}, \phi(n) \doteq n^{\prime}-n$ where $n^{\prime}$ is the largest integer in $B_{2 j+1}$.

(ii) If $n \in B_{2 j+1}$, moreover in the "first half," $\phi(n)=n^{\prime}-n$ where $n^{\prime}$ is the largest integer in $B_{2 j+1}$.

(iii) If $n \in B_{2 j+1}$, moreover in the "second half," $\phi(n)=n^{\prime}-n$ where $n^{\prime}$ is the largest integer in $B_{2 j+3}$. 
Consequently in all cases, if $n \in B_{i}, \phi(n) \geqq\left|B_{i}\right| / 2$ and hence tends to $+\infty$. Also (2.2) is immediate from the definition (2.4). Finally, $A(n, n+\phi(n))=0$ for all $n$ in case (ii), i.e. a set of asymptotic density $\frac{1}{2}\left(1-\frac{1}{2}\right)=\frac{1}{4}$.

The remainder of this section centers upon proving that for monotone $\phi$ Theorem $\mathrm{A}^{\prime}$ remains valid as stated if condition $\left({ }^{*}\right)$ is replaced by the weaker asymptotic assumption $\left({ }^{*}\right)^{\prime} A(x) \sim \alpha x$. In proving this there is no possibility of following the proof of Theorem 1 since we now have information only concerning a single sequence $A_{\phi}(n)=A(n, n+\phi(n))$, and in light of Theorem 2 the monotonicity assumption is crucial whereas it played no role in the proof of Theorem 1. Instead, a combinatorial covering argument is utilized which allows us to work with only a single $\phi$. To this end we now develop the necessary combinatorics.

Let $I \subseteq N$ and $\phi: I \rightarrow N$ be nondecreasing $\left(n_{1}<n_{2} \in I \Rightarrow \phi\left(n_{1}\right) \leqq \phi\left(n_{2}\right)\right)$. Each such $\phi$ induces a mapping $\phi^{*}: I \rightarrow N$ which is strictly increasing and defined by

$$
\phi^{*}(n)=n^{\prime}=n+\phi(n),
$$

where we shall frequently use the alternate notation $n^{\prime}$ when $\phi$ is fixed throughout an argument. More generally we shall recursively define

$$
n^{(k)}=\left(n^{(k-1)}\right)^{\prime} \quad \text { if } n^{(k-1)} \in I .
$$

Definition 1. A subset $C$ of $I$ is said to be a chain (with respect to $\phi$ ) if for each $c_{1}, c_{2} \in I$ there correspond nonnegative integers $i_{1}$ and $i_{2}$ such that $c_{1}^{\left(i_{1}\right)}=c_{2}^{\left(i_{2}\right)}$ (this implicitly requires that $c_{1}^{\left(i_{1}\right)}$ and $c_{2}^{\left(i_{2}\right)}$ be defined). $C$ is said to be a maximal chain if it is not properly included in any chain. Let $\mathscr{M}$ be the set of all maximal chains.

Comment. Maximal chains are in fact the orbits of $\phi^{*}$ on $I$.

The reader may easily verify:

LEMMA 2. (i) The maximal chains of I form a partition of $I$.

(ii) If $C \in \mathscr{M}$ and the integers of $C$ are ordered $c_{0}<c_{1}<\cdots$ we have $c_{i}=\left(c_{j}\right)^{(i-j)}$ if $i>j \geqq 0$.

(iii) $m \in I$ is the first element of some maximal chain $\Leftrightarrow m \notin I^{\prime}=\left\{n^{\prime}: n \in I\right\}$. The number of maximal chains equals $\left|I \sim I^{\prime}\right|$.

(iv) If $n \in I$, all integers in I no larger than $n$ are contained in the union of at most $\phi(n)$ maximal chains.

Definition 2. (i) For each $n \in I, I_{n}=\left(n, n^{\prime}\right] \subseteq P$.

(ii) $S \subseteq I$ is said to be a cover of $V \subseteq P$ (with respect to $\phi) \Leftrightarrow V \subseteq \bigcup_{s \in S} I_{s}$.

(iii) If $S \subseteq l$, the length of $S$ is $L(S) \doteqdot\left|\bigcup_{s \in S} I_{s}\right|$; the variation of $S$ is $|L|(S) \doteqdot$ $\sum_{s \in S}\left|I_{s}\right|$.

We shall only consider subsets $I \subseteq N$ which are segments, i.e.

$$
I=\{n \in N: x \leqq n \leqq y\}
$$

where $y=+\infty$ is admissible. In general, if $m>n$ are integers we set $I(n, m)=$ $N \cap[n, m]$ where $m=+\infty$ is admissible. 
The type of combinatorial problem we wish to consider is qualitatively stated: given two sets $E \subseteq I=I(n, m) \subseteq N$ we wish to find a cover $S \subseteq I$ of $\hat{I}=(n, m] \subseteq P$ such that

(i) $|L|(S)$ is close to $L(S)$,

(ii) $L(S \cap E)$ is large relative to $L(S)$.

Note that these two conditions are at odds with one another so that a greater precision in one necessitates a lesser precision in the other.

At first it was thought that $S$ could be taken to be the union of two chains (whose union covered $\hat{I}$ ) thereby essentially minimizing $|L|(S)-L(S)$ (condition (i)). But this would leave condition (ii) essentially uncontrollable as the following results illustrate:

Proposition 1. Given any $\delta, 0<\delta<1$, and $\varepsilon>0$ there exists an integer $n=n(\delta, \varepsilon)$, a subset $E$ of $I=I(1, n)$, and a nondecreasing function $\phi: I \rightarrow N$ such that

(i) $|E|>\delta n$,

(ii) $\phi(n)<\varepsilon n$,

(iii) every chain $C$ of I satisfies $|L|(C \cap E)=L(C \cap E)<\varepsilon n$.

Proposition 1'. To every function $w: P \rightarrow P$ tending to $+\infty$ there corresponds

1. A subset $E \subseteq N$ of asymptotic density 1 .

2. A nondecreasing $\phi: N \rightarrow N$ satisfying $\phi(n)=o(w(n))$ such that $|C \cap E|$ is finite for every chain $C$ of $N$ with respect to $\phi$.

Comment. These results are rather surprising, e.g. in the latter, 1 asserts that $E$ is essentially "all" of $N$, whereas 2 asserts that $\phi$ grows as slowly as desired, and upon partitioning $N$ into the orbits of $\phi$ on $N$ (the maximal chains) each such (infinite) orbit contains only finitely many points of $E$.

Proof. Proposition $1-$ choose $\alpha \in N$ such that

$$
1 / \alpha<\min (\varepsilon / 2,1-\delta)
$$

and set $n=n(\delta, \varepsilon)=\alpha^{\alpha}$. Define $\phi$ on $I=I(1, n)$ by

$$
\phi(k)=\alpha^{r} \quad \text { if } r \alpha^{\alpha-1} \leqq k<(r+1) \alpha^{\alpha-1} \quad(0 \leqq r<\alpha),
$$

and $\phi(n)=\alpha^{\alpha-1}$. Finally, set

$$
E=\left(\left[1, \alpha^{\alpha-1}\right] \cap N\right) \bigcup_{1 \leqq r<\alpha}\left\{n \in\left(r \alpha^{\alpha-1},(r+1) \alpha^{\alpha-1}\right]: n \not \equiv a\left(\alpha^{r}\right), \text { all } 0 \leqq a<\alpha^{r-1}\right\}
$$

then

$$
\begin{aligned}
|E|=\alpha^{\alpha-1}+\sum_{1 \leqq r<\alpha}\left(\frac{\alpha^{r}-\alpha^{r-1}}{\alpha^{r}}\right) \alpha^{\alpha-1} & =\alpha^{\alpha-1}+(\alpha-1)(1-1 / \alpha) \alpha^{\alpha-1} \geqq \alpha(1-1 / \alpha) \alpha^{\alpha-1} \\
& =\alpha^{\alpha}(1-1 / \alpha) \geqq n \delta
\end{aligned}
$$

by (2.5), and (i) of Proposition 1 is verified. Furthermore $\phi$ is clearly nondecreasing, and

$$
\phi(n)=\alpha^{\alpha-1}=(1 / \alpha) \alpha^{\alpha}=(1 / \alpha) n<(\varepsilon / 2) n
$$


by (2.5), (2.6) and (ii) of Proposition 1 is verified. Finally, by (2.6) and (2.7) every chain $C$ satisfies

$$
L(C \cap E) \leqq \alpha^{\alpha-1}+\alpha^{\alpha-1}<\varepsilon n,
$$

for if $C$ is any chain one readily verifies that if the first element of $C$ is in $\left[r \alpha^{\alpha-1},(r+1) \alpha^{\alpha-1}\right)$ then $C \cap E \subseteq\left[r \alpha^{\alpha-1},(r+1) \alpha^{\alpha-1}\right)$ and therefore

$$
L(C \cap E) \leqq \alpha^{\alpha-1}+\alpha^{\alpha-1} .
$$

The proof of Proposition 1' goes along similar lines and we merely give a sketch. Without loss of generality we may assume $w$ is monotone. Choose a sequence $\left\{l_{r}\right\}$ of positive integers tending to infinity subject to

$$
w\left(l_{1}+(2 !) l_{2}+(3 !) l_{3}+\cdots+(r !) l_{r}\right)>(r !)^{2}, \quad r \in N,
$$

and set $\alpha_{r}=l_{1}+(2 !) l_{2}+\cdots+(r !) l_{r}$ for $r \in N$. We then define $\phi: N \rightarrow N$ by

$$
\begin{aligned}
\phi(k) & =1, & & k<\alpha_{1}, \\
& =r !, & & \alpha_{r} \leqq k<\alpha_{r+1},
\end{aligned}
$$

and set

$$
E=\left(\left[1, l_{1}\right] \cap N\right) \bigcup_{r \in N}\left\{n \in\left(\alpha_{r}, \alpha_{r+1}\right]: n \not \equiv a(r !) \text { all } 0 \leqq a<(r-1) !\right\} .
$$

The verification is left to the reader.

We now prove the basic combinatorial result:

THEOREM 3. Let $\phi: I=I(n, m) \rightarrow N$ be a nondecreasing function, $0<\rho<1$, and $E \subseteq I$. Then there exists a cover $S \subseteq I$ of $\hat{I}$ such that

(i) $|L|(S)-L(S)<(1+1 /(1-\rho)) \phi(m)$,

(ii) $|L|(S \cap E)>\rho|E|$.

Proof. Let $n_{0}=n$ and define recursively for $i \geqq 1$,

$$
n_{i}=\min \left\{r \in I: \phi\left(n_{i-1}\right)<\rho \phi(r)\right\},
$$

continuing until a $n_{k}$ is determined such that $\phi\left(n_{k}\right) \geqq \rho \phi(m)$, thereupon setting $n_{k+1}=m$. We then consider the subintervals of $\hat{I}$

$$
J_{i}=\left[n_{i}, n_{i+1}\right), \quad 0 \leqq i<k, \quad J_{k}=\left[n_{k}, m\right],
$$

and set $\left|E \cap J_{i}\right|=\alpha_{i}$. Consequently

$$
\alpha_{0}+\alpha_{1}+\cdots+\alpha_{k}=|E| .
$$

Now by Lemma 2(iv) $E \cap J_{i}$ is certainly contained in the union of (the "first") $\phi\left(n_{i+1}-1\right)$ maximal chains of $I$, and consequently some maximal chain $C_{i}$ must satisfy

$$
\left|C_{i} \cap\left(E \cap J_{i}\right)\right| \geqq \alpha_{i} / \phi\left(n_{i+1}-1\right) .
$$

Let the smallest element of $C_{i} \cap J_{i}$ be $\underline{n}_{i}$ and the largest be $\bar{n}_{i}$. Note it is possible 
that $\alpha_{i}=0$ in which case $\underline{n}_{i}, \bar{n}_{i}$ are not to be defined, and in which case we call $J_{i}$ exceptional. Otherwise $\underline{n}_{i}$ and $\bar{n}_{i}$ exist (though possibly equal) and we call these $J_{i}$ regular. Finally, since successive points of $C \cap J_{i}$ are at least $\phi\left(n_{i}\right)$ apart for any chain $C$

$$
|L|\left(C_{i} \cap E \cap J_{i}\right)=L\left(C_{i} \cap E \cap J_{i}\right) \geqq\left(\alpha_{i} / \phi\left(n_{i+1}-1\right)\right) \cdot \phi\left(n_{i}\right) \geqq \rho \alpha_{i}
$$

by (2.8) and (2.10). We now construct the desired cover $S$ by filling in the "gaps" in $S_{0}=\bigcup_{1 \leqq i \leqq k}\left(C_{i} \cap J_{i}\right)$, that is adjoining points $S^{*}$ to $S_{0}$ so that $S=S^{*} \cup S_{0}$ is a cover and in so doing insuring the validity of condition (i). We proceed as follows: assume $J_{i_{1}}, J_{i_{2}}, \ldots, J_{i_{r}}$ are all the regular intervals, $i_{1}<i_{2}<\cdots<i_{r}$. First adjoin all points of the maximal chain starting at $n$ (the "first" maximal chain) which are less than $\underline{n}_{i_{1}}$; next adjoin all points $q$ of the maximal chain $C_{i_{1}}$ which satisfy $\bar{n}_{i_{1}}<q<\underline{n}_{i_{2}}$. Continue in this fashion until we adjoin all points $q$ of $C_{i_{r-1}}$ which satisfy $\bar{n}_{i_{r-1}}<q<\underline{n}_{i_{r}}$. Then finally we adjoin all points of $C_{i_{r}}$ which are greater than $\bar{n}_{i_{r}}$ and lie in $I$. The set of all these newly adjoined points is designated by $S^{*}$. By our construction $S=S^{*} \cup S_{0}$ is clearly a cover of $\hat{l}$. Moreover, by (2.9) and (2.11)

$$
\begin{aligned}
|L|(S \cap E) & =\sum_{0 \leqq i \leqq k}|L|\left(S \cap J_{i} \cap E\right) \geqq \sum_{0 \leqq i \leqq k}|L|\left(C_{i} \cap J_{i} \cap E\right) \\
& \geqq \sum_{0 \leqq i \leqq k} \rho \alpha_{i}=\rho|E|
\end{aligned}
$$

and (ii) is verified. Furthermore, by our construction each interval $J_{i}$ contains at most one overlapping pair $I_{p_{i}}$ and $I_{q_{i}}$ with $p_{i}<q_{i} \in S$ and $q_{i} \in J_{i}$. Consequently,

But by $(2.8)$,

$$
|L|(S)-L(S) \leqq \sum_{0 \leqq i \leqq k}\left|I_{p_{i}}\right|<\sum_{0 \leqq i \leqq k} \phi\left(n_{i+1}\right) .
$$

$$
\phi\left(n_{i}\right)<\rho \phi\left(n_{i+1}\right), \quad 0 \leqq i<k, \phi\left(n_{k}\right) \leqq \phi(m),
$$

which by (2.13) yields

$$
|L|(S)-L(S)<\phi(m)+\phi(m)\left(1+\rho+\cdots+\rho^{k}\right)<\left(1+\frac{1}{1-\rho}\right) \phi(m)
$$

and condition (i) is verified, completing the proof.

We are now prepared to prove:

Theorem 4. Let $A: P \rightarrow P$ be nondecreasing and set $A(u, v)=A(v)-A(u)$. Assume for some $\alpha>0$,

$$
A(x) \sim \alpha x,
$$

and let $\phi: N \rightarrow P$ be a nondecreasing function tending to $+\infty$ and such that

$$
\lim _{n \rightarrow \infty} \frac{A(n, n+\phi(n))}{\phi(n)} \leqq \alpha \quad(n \in N) .
$$

Then $A_{\phi}(n)=A(n, n+\phi(n))$ has normal order $\alpha \dot{\phi}(n)$. 
Comment. Note the special case obtained if $A=\left\{a_{v}\right\}$ is any sequence of positive real numbers tending to $+\infty$ and $A(x)=\sum_{0<a_{v} \leqq x} 1$, which was the context of Theorem $\mathrm{A}^{\prime}$ of [1], which this theorem generalizes considerably.

Proof. We shall first assume $\phi: N \rightarrow N$, and extend to the general $\phi: N \rightarrow P$ at the conclusion of the proof. Now set $A_{\phi}(n)=A(n, n+\phi(n)), n \in N$, and fix any $\varepsilon>0$. We wish to show that

$$
A^{*}(\varepsilon)=\left\{n \in N:\left|A_{\phi}(n)-\alpha \phi(n)\right|>\varepsilon \phi(n)\right\}
$$

has asymptotic density $\delta\left(A^{*}(\varepsilon)\right)=0$. By condition $\left({ }^{* *}\right)$ there is an $n_{0}=n_{0}(\varepsilon)$ such that

and consequently

$$
A_{\phi}(n)<\left(\alpha+\frac{\varepsilon}{2}\right) \phi(n) \text { for all } n \geqq n_{0}
$$

$$
A_{\phi}(n)<(\alpha-\varepsilon) \phi(n) \text { for } n \in A^{*}(\varepsilon), n \geqq n_{0} .
$$

Now fix any $\delta, 0<\delta<\varepsilon$. Then there is $n=n(\delta) \geqq n_{0}$ such that

$$
A_{\phi}(m)<(\alpha+\delta) \phi(m) \quad \text { all } m \geqq n .
$$

Now fix any $m>n$, and set

$$
I=I(n, m), \quad E=I(n, m) \cap A^{*}(\varepsilon) .
$$

Set $\rho=\frac{1}{2}$ in Theorem 3, and let $S$ be the specified cover of $I$ (we consider our initial $\phi$ now to be restricted to $I$ ). Clearly for any cover $S$

$$
\begin{aligned}
A(n, m) & \leqq \sum_{s \in S} A_{\phi}(s)=\left(\sum_{s \in S \cap E}+\sum_{s \in S \sim E}\right) A_{\phi}(s) \\
& \leqq(\alpha-\varepsilon) \sum_{s \in S \cap E} \phi(s)+(\alpha+\delta) \sum_{s \in S \sim E} \phi(s) \\
& =(\alpha-\varepsilon)|L|(S \cap E)+(\alpha+\delta)|L|(S \sim E)
\end{aligned}
$$

by (2.15) and (2.16). But as

$$
|L|(S)=|L|(S \cap E)+|L|(S \sim E), \quad|L|(S \sim E)=|L|(S)-|L|(S \cap E),
$$

and consequently,

$$
\begin{aligned}
A(n, m) & \leqq(\alpha-\varepsilon)|L|(S \cap E)+(\alpha+\delta)(|L|(S)-|L|(S \cap E)) \\
& =(\alpha+\delta)|L|(S)-(\varepsilon+\delta)|L|(S \cap E) \\
& \leqq(\alpha+\delta)(L(S)+3 \phi(m))-\frac{(\varepsilon+\delta)}{2}|E|
\end{aligned}
$$

by Theorem 3. Furthermore, since $L(S) \leqq|\hat{I}|+\phi(m)$ we finally obtain upon division by $\alpha m$

$$
\frac{A(n, m)}{\alpha m} \leqq \frac{(\alpha+\delta)}{\alpha}\left(1+\frac{4 \phi(m)}{m}\right)-\frac{(\varepsilon+\delta)}{2 \alpha} \frac{|E|}{m},
$$


and therefore by $(*)^{\prime}$

$$
1=\lim _{m \rightarrow+\infty} \frac{A(n, m)}{\alpha m} \leqq\left(1+\frac{\delta}{\alpha}\right) \lim _{m \rightarrow \infty}^{\prime} \inf \left(1+\frac{4 \phi(m)}{m}\right)-\frac{(\varepsilon+\delta)}{2 \alpha} \lim _{m \rightarrow+\infty}^{\prime} \sup _{m} \frac{|E|}{m}
$$

where the (') denotes that $m \rightarrow+\infty$ only through elements of $A^{*}(\varepsilon)$ (if $A^{*}(\varepsilon)$ is finite our assertion is trivial). We now show:

$$
\begin{aligned}
& \text { (i) } \lim _{m \rightarrow \infty} \frac{\phi(m)}{m}=0, \\
& \text { (ii) } \quad \lim _{m \rightarrow \infty}^{\prime} \sup _{m} \frac{|E|}{m}=\limsup _{m \rightarrow \infty} \frac{\left|A^{*}(\varepsilon) \cap I(1, m)\right|}{m}=\delta\left(A^{*}(\varepsilon)\right) .
\end{aligned}
$$

First, since $m \in A^{*}(\varepsilon)$,

$$
|A(m+\phi(m))-A(m)-\alpha \phi(m)|>\varepsilon \phi(m) .
$$

Also, by $\left(^{*}\right)^{\prime} \quad A(m+\phi(m))=\alpha(m+\phi(m))+o(m+\phi(m))$ and $A(m)=\alpha m+o(m)$, yielding $o(m+\phi(m))+o(m)>\varepsilon \phi(m)$. This in turn implies

$$
o\left(1+\frac{\phi(m)}{m}\right)+o(1)>\varepsilon \frac{\phi(m)}{m}
$$

i.e.

$$
o\left(\frac{\phi(m)}{m}\right)+o(1)>\varepsilon \frac{\phi(m)}{m}
$$

as $m \in A^{*}(\varepsilon) \rightarrow+\infty$, which clearly implies (i). To prove (ii), note that $(n=n(\delta)$ is fixed)

$$
\lim _{m \rightarrow+\infty}^{\prime} \frac{|E|}{\left|A^{*}(\varepsilon) \cap I(1, m)\right|}=\lim _{m \rightarrow \infty} \frac{\left|A^{*}(\varepsilon) \cap I(n, m)\right|}{\left|A^{*}(\varepsilon) \cap I(1, m)\right|}=1,
$$

which implies

$$
\lim _{m \rightarrow \infty}^{\prime} \sup \frac{|E|}{m}=\lim _{m \rightarrow \infty}^{\prime} \sup \frac{\left|A^{*}(\varepsilon) \cap I(1, m)\right|}{m}=\delta\left(A^{*}(\varepsilon)\right),
$$

as asserted, since the upper asymptotic density $\delta\left(A^{*}(\varepsilon)\right)$ of $A^{*}(\varepsilon)$ is clearly obtained by taking the limit through elements of $A^{*}(\varepsilon)$. Thus (2.21) yields, in light of (2.22),

$$
1 \leqq 1+\frac{\delta}{\alpha}-\left(\frac{\varepsilon+\delta}{2 \alpha}\right) \delta\left(A^{*}(\varepsilon)\right)
$$

which simplifies to

$$
\delta\left(A^{*}(\varepsilon)\right) \leqq \frac{\delta}{\alpha}\left(\frac{2 \alpha}{\varepsilon+\delta}\right)=\frac{2 \delta}{\varepsilon+\delta}
$$

The theorem now follows if $\delta \rightarrow 0^{+}$(under the assumption that $\phi: N \rightarrow N$ ).

Now in general given any nondecreasing $\phi: N \rightarrow P$ (since the theorem is in fact an asymptotic statement we may without loss of generality assume $\phi \geqq 1), \delta(n)=$ $[\phi(n)]$ is a nondecreasing function and $\tilde{\phi}: N \rightarrow N$. One easily verifies $(* *)$ for $\tilde{\phi}$, 
since $\tilde{\phi} \rightarrow+\infty$ and $A_{\tilde{\phi}} \leqq A_{\phi}$. Consequently by what we have just proved $A_{\tilde{\phi}}$ has normal order $\alpha \tilde{\phi} \sim \alpha \phi$, and a final application of $\left({ }^{* *}\right)$ to $A_{\phi} \geqq A_{\tilde{\phi}}$ yields the theorem in general.

III. Generalizations to other asymptotic distributions. The question now arises as to which functions $f(x)$ other than linear $f(x)=\alpha x, \alpha>0$, yield an analogue of Theorem 4 if $\left({ }^{*}\right)^{\prime}$ is replaced by $A(x) \sim f(x)$. In this section we show that our combinatorial theorem may be generalized to prove an analogue of Theorem 4 for any function $f(x)$ with nondecreasing derivative $f^{\prime}(x)$. In intuitive terms this means any function $f$ with at least a linear order of growth. We then show by counterexample that for the function $f(x)=\log x$ (of "very slow" order of growth) the analogue of Theorem 4 is not valid. Our techniques can be extended to other functions, but still the question of simple necessary and sufficient conditions on a function $f$ to satisfy an analogue of Theorem 4 is open.

The main tool required for our generalization is an analogue of our combinatorial Theorem 3. We first develop some new notation:

Definition 3. (Notation as in Definition 2 and preceding.) Let $f: N \rightarrow P$ be nondecreasing, then

(i) $\left|L_{f}\right|(S)=\sum_{s \in S}\left(f\left(s^{\prime}\right)-f(s)\right) \doteqdot \sum_{s \in S} f_{\phi}(s)$,

(ii) $L_{f}(S)=\int_{U_{s \in S} I_{s}} d f(s)$.

Comment. The definition of $L$ and $|L|$ in §II corresponds to the special case $f(x)=x$, and we see that in some sense our generalization bears the same relation to the initial case as the Riemann-Stieltjes integral does to the Riemann integral. Finally note that the definition in (ii) simply states that if $\bigcup_{s \in S} I_{s}$ is written as the disjoint union of intervals

$$
\bigcup_{s \in S} I_{s}=\bigcup_{i}\left(\alpha_{i}, \beta_{i}\right]
$$

then $L_{f}(S)=\sum_{i} f\left(\beta_{i}\right)-f\left(\alpha_{i}\right)$.

We now prove

THEOREM 5. Let $\phi: I=I(n, m) \rightarrow N$ be a nondecreasing function, $0<\rho<1$, and $E \subseteq I$. Furthermore let $f:[n, m] \rightarrow P$ have a nondecreasing derivative $f^{\prime}>0$. Then, if $|E| \geqq w|\hat{I}|>0$ and $0 \leqq \theta<w$ there exists a subinterval $\tilde{I}=\{n, n+1, \ldots, \tilde{m}\} \subseteq I$ with $\tilde{m} \in E$ and a cover $\tilde{S}$ of $\tilde{I}, \tilde{m} \in \tilde{S}$, such that

(i) $|\tilde{I}|>\theta|\hat{I}|$,

(ii) $\left|L_{f}\right|(\tilde{S})-L_{f}(\tilde{S})<(1+1 /(1-\rho)) f(\tilde{m})$,

(iii) $\left|L_{f}\right|(\tilde{S} \cap E)>\rho(w-\theta)(f(\tilde{m})-f(n))=\rho(w-\theta) f(n, \tilde{m})$.

Proof. We set $f_{\phi}(r)=f(r+\phi(r))-f(r)$, and note that $f_{\phi}$ is a nondecreasing function since $\phi$ and $f^{\prime}$ are nondecreasing. Set $n_{0}=n$, and define recursively for $i \geqq 1$

$$
n_{i}=\min \left\{r \in I: f_{\phi}\left(n_{i-1}\right)<\rho f_{\phi}(r)\right\}
$$


continuing until a $n_{k}$ is determined such that $f_{\phi}\left(n_{k}\right) \geqq \rho f_{\phi}(m)$, thereupon setting $n_{k+1}=m$. We then consider the subintervals of $I$

$$
J_{i}=\left[n_{i}, n_{i+1}\right), \quad 0 \leqq i<k, \quad J_{k}=\left[n_{k}, m\right],
$$

and set $\left|E \cap J_{i}\right|=\alpha_{i}$. Consequently,

$$
\alpha_{0}+\alpha_{1}+\cdots+\alpha_{k}=|E| \geqq w|\hat{I}| .
$$

Now by Lemma 2(iv) $E \cap J_{i}$ is certainly contained in the union of $\phi\left(n_{i}^{*}\right)$ chains of $I$ where $n_{i}^{*}$ is the largest integer in $J_{i}\left(n_{i}^{*}=n_{i+1}-1\right.$ if $0 \leqq i<k, n_{i}^{*}=m$ if $\left.i=k\right)$, and thus for some chain $C_{i}$

$$
\left|C_{i} \cap E \cap J_{i}\right| \geqq \alpha_{i} / \phi\left(n_{i}^{*}\right) .
$$

Consequently, by (3.1) and the monotonicity of $f_{\phi}$

$$
\begin{aligned}
\left|L_{f}\right|\left(C_{i} \cap E \cap J_{i}\right) & =L_{f}\left(C_{i} \cap E \cap J_{i}\right) \geqq\left(\frac{\alpha_{i}}{\phi\left(n_{i}^{*}\right)}\right) f_{\phi}\left(n_{i}\right) \geqq \rho \alpha_{i}\left(\frac{f_{\phi}\left(n_{i}^{*}\right)}{\phi\left(n_{i}^{*}\right)}\right) \\
& \geqq \rho \alpha_{i}\left(\frac{f\left(n_{i+1}\right)-f\left(n_{i}\right)}{n_{i+1}-n_{i}}\right) .
\end{aligned}
$$

Now complete $S_{0}=\bigcup_{0 \leqq i \leqq k}\left(C_{i} \cap J_{i}\right)$ to a cover $S$ of $I$ exactly as done in the proof of Theorem 3. Now for $0 \leqq i \leqq k$ set

$$
X_{i}=\frac{f\left(n_{i+1}\right)-f\left(n_{i}\right)}{n_{i+1}-n_{i}}=\frac{f\left(n_{i}, n_{i+1}\right)}{\left|J_{i}\right|}
$$

and note that $X_{i} \leqq X_{i+1}$ for $0 \leqq i<k$ since $f^{\prime}$ is nondecreasing. Now for any $r$, $0 \leqq r \leqq k$ consider

$$
\sum_{r}=\sum_{0 \leqq i \leqq r} L_{f}\left(C_{i} \cap E \cap J_{i}\right)
$$

Then by (3.4) and (3.5)

$$
\sum_{r} \geqq \rho\left(\sum_{0 \leqq i \leqq r} \alpha_{i} X_{i}\right)
$$

Let $r_{0} \geqq 0$ be the smallest integer such that

$$
\sum_{0 \leqq i \leqq r} \alpha_{i} \geqq \theta|\hat{I}|+(w-\theta) \sum_{0 \leqq i \leqq r}\left|J_{i}\right|,
$$

which certainly exists since $r=k$ satisfies (3.8) by virtue of (3.2). Note further that $\alpha_{r_{0}}>0$ necessarily and consequently $C_{r_{0}} \cap E \cap J_{r_{0}} \neq \varnothing$. Claim we may take $\tilde{m}$ to be the largest element of $C_{r_{0}} \cap E \cap J_{r_{0}}$ and $\tilde{S}=S \cap \tilde{I}$ (note that $\tilde{m}$ is therefore the largest element of $\tilde{S}$ ). It remains to verify conditions (i)-(iii):

First (i) is immediately verified by virtue of (3.8), and the proof of (ii) is identical to the proof of the corresponding assertion (i) of Theorem 3 where (3.1) plays the role of (2.8). Now to prove (iii) we first set

$$
\Delta_{i}=\alpha_{i}-(w-\theta)\left|J_{i}\right|, \quad 0 \leqq i \leqq k,
$$


and note that by (3.5)

$$
f(\tilde{m})-f(n)=\sum_{0 \leqq i<r_{0}}\left|J_{i}\right| X_{i}+\left(f(\tilde{m})-f\left(n_{r_{0}}\right)\right) \leqq \sum_{0 \leqq i \leqq r_{0}}\left|J_{i}\right| X_{i} .
$$

Now by (3.6) and (3.7)

$$
\left|L_{f}\right|(\tilde{S} \cap E) \geqq \sum_{0 \leqq i \leqq r_{0}} L_{f}\left(C_{i} \cap E \cap J_{i}\right) \geqq \rho \sum_{0 \leqq j \leqq r_{0}} \alpha_{i} X_{i},
$$

and consequently by (3.9)

$$
\left|L_{f}\right|(\tilde{S} \cap E)-(w-\theta) \rho(f(\tilde{m})-f(n)) \geqq \rho \sum_{0 \leqq i \leqq r_{0}} \Delta_{i} X_{i} .
$$

Now since $X_{i} \leqq X_{i+1}$ we may introduce new nonnegative parameters $Y_{i}$ defined by

$$
X_{i}=Y_{0}+Y_{1}+\cdots+Y_{i}, \quad 0 \leqq i \leqq k .
$$

Furthermore, by definit:on of $r_{0}$

$$
\sum_{0 \leqq i \leqq r} \Delta_{i}<\theta|\hat{I}| \text { for all } r<r_{0}
$$

whereas

$$
\sum_{0 \leqq i \leqq r_{0}} \Delta_{i} \geqq \theta|\hat{I}|
$$

which implies

$$
\sum_{r \leqq i \leqq r_{0}} \Delta_{i}=\sum_{0 \leqq i \leqq r_{0}} \Delta_{i}-\sum_{0 \leqq i \leqq r} \Delta_{i} \geqq 0 \text { for all } r \leqq r_{0} .
$$

Therefore,

$$
\sum_{0 \leqq i \leqq r_{0}} \Delta_{i} X_{i}=\sum_{0 \leqq r \leqq r_{0}} Y_{r}\left(\sum_{r \leqq i \leqq r_{0}} \Delta_{i}\right) \geqq 0
$$

by (3.12) and (3.15), which concludes the proof of (iii) in light of (3.11).

Comment. Note that Theorem 3 corresponds to the special case $f(x)=x$ and $\theta=0$, and states that in this case we may always in fact take $\tilde{I}=I$, i.e. $\tilde{m}=m$. The reason for the necessity of choosing a subinterval $\tilde{I}$ in general in Theorem 5 as opposed to the simple situation in Theorem 3 is the nonuniformity of the growth of $f$ in general, e.g. if $E$ is concentrated in the "beginning" of $I$ and $f$ grows very rapidly. Furthermore a condition such as (i) must be appended for in the application we must guarantee that $\tilde{I}$ is not "too small" relative to $I$.

We now use Theorem 5 to prove the promised generalization of Theorem 4 in much the same way as Theorem 3 was used to prove Theorem 4.

Theorem 6. Let $A: P \rightarrow P$ be nondecreasing and set $A(u, v)=A(v)-A(u)$. Assume that

$$
A(x) \sim f(x)
$$


where $f: P \rightarrow P$ has nondecreasing derivative $f^{\prime}>0$. Furthermore, let $\phi: N \rightarrow P$ be a nondecreasing function tending to $+\infty$ and such that

$$
\limsup _{n \rightarrow \infty} \frac{A(n, n+\phi(n))}{f(n, n+\phi(n))} \leqq 1 \quad(n \in N),
$$

where $f(n, n+\phi(n))=f(n+\phi(n))-f(n)$. Then $A_{\phi}(n)=A(n, n+\phi(n))$ has normal order $f_{\phi}(n)=f(n, n+\phi(n))$.

Proof. We follow the proof of Theorem 4: first assume $\phi: N \rightarrow N$, and fix any $\varepsilon>0$. We wish to show that

$$
A^{*}(\varepsilon)=\left\{n \in N:\left|A_{\phi}(n)-f_{\phi}(n)\right|>\varepsilon f_{\phi}(n)\right\}
$$

has asymptotic density $\delta\left(A^{*}(\varepsilon)\right)=0$. We give a proof by contradiction, assuming

$$
\bar{\delta}\left(A^{*}(\varepsilon)\right)=\limsup _{m \rightarrow \infty} \frac{\left|A^{*}(\varepsilon) \cap I(1, m)\right|}{m}=2 w>0 .
$$

By condition $\left({ }^{* *}\right)^{\prime}$ there is an $n_{0}=n_{0}(\varepsilon)$ such that

$$
A_{\phi}(n)<\left(1+\frac{\varepsilon}{2}\right) f_{\phi}(n) \text { for all } n \geqq n_{0},
$$

and consequently

$$
A_{\phi}(n)<(1-\varepsilon) f_{\phi}(n) \text { if } n \in A^{*}(\varepsilon), n \geqq n_{0} .
$$

Now fix any $\delta, 0<\delta<\varepsilon$. Then again by $\left({ }^{* *}\right)^{\prime}$ there is a $n=n(\delta) \geqq n_{0}$ such that

$$
A_{\phi}(m) \leqq(1+\delta) f_{\phi}(n) \text { for all } m \geqq n \text {. }
$$

Now for any $m>n$, set

$$
I=I(n, m), \quad E=I(n, m) \cap A^{*}(\varepsilon) .
$$

Now by (3.16) (for this fixed value of $n=n(\delta)$ ) we may certainly find a subsequence $m_{i} \rightarrow+\infty$ for which $|E| \geqq w|\hat{I}|=w(m-n)$, and we may assume each such $m_{i} \in A^{*}(\varepsilon)$. We now apply Theorem 5 with $I_{i}=I\left(n, m_{i}\right), E_{i}=I\left(n, m_{i}\right) \cap A^{*}(\varepsilon), \rho=\frac{1}{2}$ and $\theta=w / 2$. Let $\tilde{I}_{i}, \tilde{m}_{i}$, and $\tilde{S}_{i}$ be as guaranteed by Theorem 5 . Then,

$$
\begin{aligned}
A\left(n, \tilde{m}_{i}\right) & \leqq A\left(n, \tilde{m}_{i}+\phi\left(\tilde{m}_{i}\right)\right) \leqq \sum_{s \in \tilde{S_{i}}} A_{\phi}(s) \leqq \sum_{s \in \tilde{S_{i}} \cap E_{i}} A_{\phi}(s)+\sum_{s \in \tilde{S_{i}} \sim E_{i}} A_{\phi}(s) \\
& \leqq(1-\varepsilon)\left|L_{f}\right|\left(\tilde{S}_{i} \cap E_{i}\right)+(1+\delta)\left|L_{f}\right|\left(\tilde{S_{i}} \sim E_{i}\right)
\end{aligned}
$$

by (3.17), (3.18) and Definition 3(i). Furthermore,

$$
\left|L_{f}\right|\left(\tilde{S_{i}}\right)=\left|L_{f}\right|\left(\tilde{S}_{i} \cap E_{i}\right)+\left|L_{f}\right|\left(\tilde{S}_{i} \sim E_{i}\right)
$$

implies by Theorem 5(ii)

$$
\left|L_{f}\right|\left(\tilde{S}_{i} \sim E_{i}\right)<L_{f}(\tilde{S})+3 f_{\phi}(\tilde{m})-\left|L_{f}\right|\left(\tilde{S}_{i} \cap E_{i}\right),
$$


which upon substitution into (3.20) yields

$$
\begin{aligned}
A\left(n, \tilde{m}_{i}\right) & \leqq(1-\varepsilon)\left|L_{f}\right|\left(\tilde{S}_{i} \cap E_{i}\right)+(1+\delta)\left(L_{f}\left(\tilde{S}_{i}\right)+3 f_{\phi}\left(\tilde{m}_{i}\right)-\left|L_{f}\right|\left(\tilde{S}_{i} \cap E_{i}\right)\right) \\
& =(1+\delta)\left(L_{f}\left(\tilde{S}_{i}\right)+3 f_{\phi}\left(\tilde{m}_{i}\right)\right)-(\varepsilon+\delta)\left|L_{f}\right|\left(\tilde{S}_{i} \cap E_{i}\right) .
\end{aligned}
$$

However, by Theorem 5 ,

$$
\begin{gathered}
L_{f}\left(\tilde{S_{i}}\right)=f\left(n, \tilde{m}_{i}+\phi\left(\tilde{m}_{i}\right)\right)=f\left(n, \tilde{m}_{i}\right)+f_{\phi}\left(\tilde{m}_{i}\right), \\
\left|L_{f}\right|\left(\tilde{S}_{i} \cap E_{i}\right)>\frac{w}{4} f\left(n, \tilde{m}_{i}\right),
\end{gathered}
$$

which upon substitution in (3.22) yields

$$
A\left(n, \tilde{m}_{i}\right) \leqq(1+\delta)\left(f\left(n, \tilde{m}_{i}\right)+4 f_{\phi}\left(\tilde{m}_{i}\right)\right)-\frac{(\varepsilon+\delta) w}{4} \cdot f\left(n, \tilde{m}_{i}\right)
$$

Furthermore, by Theorem 5(i) as $m_{i} \rightarrow+\infty$, so does $\tilde{m}_{i} \in E_{i} \subseteq A^{*}(\varepsilon)$. Consequently (since $n=n(\delta)$ is temporarily fixed),

$$
\lim _{i \rightarrow+\infty} A\left(n, \tilde{m}_{i}\right) \sim A\left(\tilde{m}_{i}\right), \quad \lim _{i \rightarrow+\infty} f\left(n, \tilde{m}_{i}\right) \sim f\left(\tilde{m}_{i}\right),
$$

and thus (3.23) implies

$$
\begin{aligned}
1 & =\lim _{i \rightarrow \infty} \frac{A\left(\tilde{m}_{i}\right)}{f\left(\tilde{m}_{i}\right)} \leqq \limsup _{i \rightarrow \infty}\left\{(1+\delta)\left(1+\frac{4 f_{\phi}\left(\tilde{m}_{i}\right)}{f\left(\tilde{m}_{i}\right)}\right)-\frac{(\varepsilon+\delta) w}{4}\right\} \\
& =1+\delta-\left(\frac{\varepsilon+\delta}{4}\right) w+4 \limsup _{i \rightarrow \infty} \frac{f_{\phi}\left(\tilde{m}_{i}\right)}{f\left(\tilde{m}_{i}\right)}
\end{aligned}
$$

by $\left({ }^{*}\right) "$.

But since $\tilde{m}_{i} \in A^{*}(\varepsilon) \rightarrow+\infty$,

$$
\lim _{i \rightarrow \infty} \frac{f_{\phi}\left(\tilde{m}_{i}\right)}{f\left(\tilde{m}_{i}\right)}=0:
$$

For $\left|A_{\phi}\left(\tilde{m}_{i}\right)-f_{\phi}\left(\tilde{m}_{i}\right)\right|>\varepsilon f_{\phi}\left(\tilde{m}_{i}\right)$ implies

$$
\left|A\left(\tilde{m}_{i}+\phi\left(\tilde{m}_{i}\right)\right)-A\left(\tilde{m}_{i}\right)+f\left(\tilde{m}_{i}\right)-f\left(\tilde{m}_{i}+\phi\left(\tilde{m}_{i}\right)\right)\right|>\varepsilon f_{\phi}\left(\tilde{m}_{i}\right),
$$

which implies by $\left({ }^{*}\right)^{\prime}$

$$
\begin{aligned}
\varepsilon f_{\phi}\left(\tilde{m}_{i}\right) & <\left|A\left(\tilde{m}_{i}+\phi\left(\tilde{m}_{i}\right)\right)-f\left(\tilde{m}_{i}+\phi\left(\tilde{m}_{i}\right)\right)\right|+\left|A\left(\tilde{m}_{i}\right)-f\left(\tilde{m}_{i}\right)\right| \\
& =o\left(f\left(\tilde{m}_{i}+\phi\left(\tilde{m}_{i}\right)\right)\right)+o\left(f\left(\tilde{m}_{i}\right)\right)=o\left(f\left(\tilde{m}_{i}\right)+f_{\phi}\left(\tilde{m}_{i}\right)\right),
\end{aligned}
$$

or upon setting $q_{i}=f_{\phi}\left(\tilde{m}_{i}\right) / f\left(\tilde{m}_{i}\right), \varepsilon q_{i}=o(1)+o\left(q_{i}\right)$, which clearly implies $q_{i} \rightarrow 0$ and hence (3.26). Returning to (3.25) we therefore have $1 \leqq 1+\delta-((\varepsilon+\delta) / 4) w$ or

$$
w \leqq 4 \delta / \varepsilon+\delta .
$$

But (3.27) must be true for all $\delta, 0<\delta<\varepsilon$, which implies $w=0$, a contradiction. 
Now considering the general case of nondecreasing $\phi: N \rightarrow P$, we see that the argument at the end of the proof of Theorem 3 which justifies the extension in case $f(x)=\alpha x$ is linear may not be used here for it depended on

$$
\lim _{n \rightarrow \infty} \frac{f(n, n+[\phi(n)])}{f(n, n+\phi(n))}=1,
$$

which is clearly valid for linear $f$ but not for all functions $f$ presently considered. Nevertheless, a proof may be given along the following lines, the details being left to the reader: if $\phi: I \rightarrow P$ is nondecreasing (once more we may assume $\phi \geqq 1$ for the technical purposes of proving the theorem), first define the nondecreasing function $\phi: I \rightarrow N$ by $\phi(n)=[\phi(n)]$. Then define the chains of $\phi$ on $I$ to be simply the chains of $\tilde{\phi}$ on $I$, noting in this case that if $r$ and $s$ are consecutive points of a chain of $\phi,(r, r+\phi(r)]$ and $(s, s+\phi(s)]$ may overlap, but only in a region of length at most one. Next extend Theorem 5 to general nondecreasing $\phi: I \rightarrow P(\phi \geqq 1)$ by essentially duplicating the proof and using the above notion of a chain of $\phi$ (Definitions 2 and 3 have their obvious extension to nonintegral $\phi$ ). The only difference in this extended version of Theorem 5 is in the estimate (ii) in which an extra term must be added to the right to account for the "overlap" even in the individual chains $C_{i}$ themselves (as opposed to the automatic disjointness of the $I_{r}$ in the chains for integral $\phi$ ). But this is essentially estimated above by

$$
(2 / \phi(n)) f(n, \tilde{m}) \text {. }
$$

The proof of Theorem 6 in the general case now follows just as the integer valued case given already with only a minor modification to allow for the new term (3.28) in the application of Theorem 5 (modified as above).

To prove $f(x)=\log x$ does not satisfy a result analogous to Theorem 6 , set $A=\left\{e^{n}: n \in N, n \neq m^{2}+1, m \in N\right\}$ and also set $A(x)=\sum_{a_{v} \leqq x ; a_{v} \in A}$ 1. Furthermore let $\phi(n)=(e-1) n$. Then $\left({ }^{*}\right)^{\prime \prime}$ and $\left({ }^{* *}\right)^{\prime \prime}$ of Theorem 6 are easily verified while $A_{\phi}(n)=$ $A(e n)-A(n)$ does not have normal order $f_{\phi}(n) \equiv 1$ since $A_{\phi}(n)=0$ for $\exp m^{2}<n$ $\leqq e \cdot \exp m^{2}$ and $m \in N$. Further modifications of this example yield the same result for appropriate $A$ and $\phi$ for which $f_{\phi} \uparrow+\infty$.

IV. Continuous analogues. All the results of $\S$ II and III have continuous analogues, and the purpose of this section is to give the requisite definitions and sketch the proofs of the theorems related to those in II. The results of §III may be similarly carried out.

Definition 4. Let $g, f: P \rightarrow P$ be Lebesgue measurable. We then say $g$ has normal order $f$ iff for every $\varepsilon>0$

$$
\lim _{x \rightarrow \infty} \frac{|\{y<x, y \in P:|g(y)-f(y)|>\varepsilon f(y)\}|}{x}=0 .
$$

Let $\phi: P \rightarrow P$ be nondecreasing. Each such $\phi$ induces a mapping $\phi^{*}: P \rightarrow P$ which is strictly increasing and given by $\phi^{*}(x)=x+\phi(x), x \in P$. Once $\phi$ is fixed we 
shall use the alternate notation $x^{\prime}=x+\phi(x)$, and more generally $x^{(k)}=\left(x^{(k-1)}\right)^{\prime}$ for $k=2,3, \ldots$

Definition 5. A subset $C$ of $P$ is said to be a chain (with respect to $\phi$ ) $\Leftrightarrow$ for each $x, y \in C$ there correspond $i, j \in N$ such that $x^{(i)}=y^{(j)}$. $C$ is said to be a maximal chain if it is not properly included in any chain.

Comment. As in the discrete case the maximal chains are simply the orbits of $\phi^{*}$ on $P$, and the maximal chains give a partition of $P$.

Definition 6. For $I \subseteq P$ we say $S \subseteq P$ is a cover of $I$ (with respect to $\phi) \Leftrightarrow$

$$
I \subseteq \bigcup_{s \in S} I_{s}=\bigcup_{s \in S}\left(s, s^{\prime}\right]
$$

Furthermore, we define

$$
|L|(S) \doteqdot \sum_{s \in S}\left|I_{s}\right| \leqq+\infty, \quad L(S) \doteqdot\left|\bigcup_{s \in S} I_{s}\right| .
$$

We now wish to prove an analogue of the basic combinatorial covering resultTheorem 3. But before doing so we require a simple measure theoretic result which was not needed for the "discrete" calculations of II.

Lemma 3. Let $X$ be a measurable subset of $P$, and set $X^{(i)}=\left\{x^{(i)}: x \in X\right\}$. Then $X^{(i)}$ is measurable and $\left|X^{(i)}\right| \geqq|X|$ for all $i \in N$.

Proof. It clearly suffices to consider $i=1$, by induction. First

$$
X^{\prime}=\{x+\phi(x): x \in X\}
$$

is clearly measurable since $\phi^{*}$ is monotone. Now let $\phi=\phi_{c}+\phi_{s}$ be the decomposition of $\phi$ into its monotone continuous $\left(\phi_{c}\right)$ and jump $\left(\phi_{s}\right)$ components. Then $\phi^{*}(x)=$ $\phi_{c}^{*}+\phi_{s}$ is the corresponding decomposition of $\phi^{*}$, where $\phi_{c}^{*}(x)=x+\phi_{c}(x)$. Clearly the measure of $X^{\prime}=\phi^{*}(X)$ is the same as that of $\phi_{c}^{*}(X)$. But by 11.54 of [2],

$$
\left|X^{\prime}\right|=\left|\phi_{c}^{*}(X)\right| \geqq \int_{X}\left(\phi_{c}^{*}(x)\right)^{\prime} d x=\int_{X}\left(1+\phi_{c}^{\prime}(x)\right) d x \geqq|X| .
$$

We are now ready to prove

THEOREM 7. Let $\phi: I=[x, y] \rightarrow P$ be a nondecreasing function, $0<\rho<1$, and $E \subseteq I$ be a measurable subset. Then there exists a (finite) cover $S \subseteq I$ of $(x, y]$ such that

(i) $|L|(S)-L(S)<(1+1 /(1-\rho)) \phi(y)$,

(ii) $|L|(S \cap E)>\rho|E|$.

Proof. Let $x_{0}=x$, and define recursively for $i \geqq 1$

$$
x_{i}=\inf \left\{z \in I: \phi\left(x_{i-1}\right)<\rho \phi(z)\right\}
$$

continuing until a $x_{k}$ is determined such that $\phi\left(x_{k}\right) \geqq \rho \phi(y)$, thereupon setting $x_{k+1}=y$. We then consider the subintervals of $I$.

$$
J_{i}=\left[x_{i}, x_{i+1}\right), \quad 0 \leqq i<k, \quad J_{k}=\left[x_{k}, y\right],
$$


and set $\left|E \cap J_{i}\right|=\alpha_{i}$. Now consider the iterates,

$$
x_{i}^{(0)}=x_{i}, x_{i}^{(1)}, x_{i}^{(2)}, \ldots, x_{i}^{(r)}<x_{i+1} \leqq x_{i}^{(r+1)} \quad(r=r(i)),
$$

and the subintervals

$$
J_{i}^{j}=\left[x_{i}^{(j)}, x_{i}^{(j+1)}\right), \quad 0 \leqq j<r, \quad J_{i}^{r}=\left[x_{i}^{(r)}, x_{i+1}\right) .
$$

Clearly $\alpha_{i}=\sum_{0 \leqq j \leqq r}\left|E \cap J_{i}^{j}\right|$, and consequently by Lemma 3,

$$
\alpha_{i} \leqq \sum_{0 \leqq j \leqq r}\left|\left(E \cap J_{i}^{j}\right)^{(r-j)}\right|, \quad\left(E \cap J_{i}^{j}\right)^{(r-j)} \subseteq\left[x_{i}^{(r)}, x_{i}^{(r+1)}\right) .
$$

Now assume all maximal chains $C$ of $I$ satisfy $\left|C \cap E \cap J_{i}\right| \leqq w$. This implies that each point of $\left[x_{i}^{(r)}, x_{i}^{(r+1)}\right)$ is contained in at most $w$ of $\left(E \cap J_{i}^{j}\right)^{(r-j)}(0 \leqq j \leqq r)$, and consequently

$$
\sum_{0 \leqq j \leqq r}\left|\left(E \cap J_{i}^{j}\right)^{(r-j)}\right| \leqq w\left(x_{i}^{(r+1)}-x_{i}^{(r)}\right)=w \phi\left(x_{i}^{(r)}\right) .
$$

Therefore (4.5) and (4.6) show that for some maximal chain $C_{i}$

$$
\left|C_{i} \cap E \cap J_{i}\right| \geqq \alpha_{i} / \phi\left(x_{i}^{(r)}\right) \quad\left(x_{i}^{(r)}<x_{i+1}\right) .
$$

From this point on the proof is the same as that for Theorem 3.

Reasoning just as in the proof of Theorem 3 (in light of Theorem 7 just proved) the following analogue of Theorem 4 may be proved, the proof being left to the reader.

TheOREM 8. Let $A: P \rightarrow P$ be nondecreasing and set $A(u, v)=A(v)-A(u)$. Assume for some $\alpha>0$,

$$
A(x) \sim \alpha x
$$

and let $\phi: P \rightarrow P$ be a nondecreasing function tending to $+\infty$ and such that

$$
\limsup _{x \rightarrow+\infty} \frac{A(x, x+\phi(x))}{\phi(x)} \leqq \alpha \quad(x \in P) .
$$

Then $A_{\phi}(x)=A(x, x+\phi(x))$ has normal order $\alpha \phi(x)$.

\section{BIBLIOGRAPHY}

1. R. Bellman and H. N. Shapiro, The distribution of squarefree integers in small intervals, Duke Math. J. 21 (1954), 629-637.

2. E. C. Titchmarsh, The theory of functions, Oxford Univ. Press, Oxford, 1939.

Courant Institute, New York University, New York, New YorK 